\title{
Erratum to: Interaction integrals for fracture analysis of functionally graded magnetoelectroelastic materials
}

\author{
B. N. Rao - M. Kuna
}

Published online: 19 January 2010

(C) Springer Science+Business Media B.V. 2010

\section{Erratum to: Int J Fract (2008) 153:15-37 DOI 10.1007/s10704-008-9285-4}

The authors regret that unfortunately an erroneous version of the Appendix to their article was published. Please find below the correct Appendix that should be regarded by the reader as the final version

\section{Appendix}

Proof of existence of interaction integral for FGMMs

Equations 56, 60 and 64 contains the second integral involving extra terms due to material nonhomogeneity. The existence of the second integral in Eqs. 56, 60 and 64 , as the limit $r \rightarrow 0$ is proved below. Since, spatial derivatives of the material properties, $C_{i j k s}, e_{s i j}, h_{s i j}$, $\kappa_{i s}, \beta_{i s}, \gamma_{i s}$, are bounded at the crack tip, i.e., $C_{i j k s, 1}$,

The online version of the original article can be found under doi:10.1007/s10704-008-9285-4.

B. N. Rao $(\varangle)$

Structural Engineering Division, Department of Civil Engineering, Indian Institute of Technology, Madras, Chennai 600036, India

e-mail: bnrao@iitm.ac.in

M. Kuna

TU Bergakademie Freiberg, Institut für Mechanik und Fluiddynamik, Lampadiusstraße 4, 09596 Freiberg, Germany $e_{s i j, 1}, h_{s i j, 1}, \kappa_{i s, 1}, \beta_{i s, 1}$ and $\gamma_{i s, 1}$ are $O\left(r^{\alpha}\right)$ with $\alpha \geq$ 0 , the second integral in Eqs. 56, 60 and 64 exists as the limit $r \rightarrow 0$. In the limit $r \rightarrow 0, C_{i j k s}(r, \theta)=$ $C_{i j k s}^{*}, e_{s i j}(r, \theta)=e_{s i j}^{*}, h_{s i j}(r, \theta)=h_{s i j}^{*}, \kappa_{i s}(r, \theta)=$ $\kappa_{i s}^{*}, \beta_{i s}(r, \theta)=\beta_{i s}^{*}$ and $\gamma_{i s}(r, \theta)=\gamma_{i s}^{*}$ in Eq. 7. Hence in the limit $r \rightarrow 0$, in constant constitutive tensor formulation $\sigma_{i j}^{(1)} \varepsilon_{i j, 1}^{(2)}=\sigma_{i j, 1}^{(2)} \varepsilon_{i j}^{(1)}, \sigma_{i j, 1}^{(1)} \varepsilon_{i j}^{(2)}=\sigma_{i j}^{(2)} \varepsilon_{i j, 1}^{(1)}$, $D_{j}^{(1)} E_{j, 1}^{(2)}=D_{j, 1}^{(2)} E_{j}^{(1)}, D_{j, 1}^{(1)} E_{j}^{(2)}=D_{j}^{(2)} E_{j, 1}^{(1)}, B_{j}^{(1)}$ $H_{j, 1}^{(2)}=B_{j, 1}^{(2)} H_{j}^{(1)}$, and $B_{j, 1}^{(1)} H_{j}^{(2)}=B_{j}^{(2)} H_{j, 1}^{(1)}$, and in incompatibility formulation $u_{i, j 1}^{(2)}=\varepsilon_{i j, 1}^{(2)}, \phi_{, j 1}^{(2)}=$ $-E_{j, 1}^{(2)}$, and $\varphi_{, j 1}^{(2)}=-H_{j, 1}^{(2)}$. Therefore, the integrand of the second integral in Eq. 56 and the incompatibility terms in the integrand of the second integral in Eq. 60 naturally vanish. Note that in incompatibility formulation the auxiliary fields are compatible very near the crack tip (asymptotically).

(i) In addition, since in constant constitutive tensor formulation the auxiliary stress, electrical displacement, and magnetic induction are related to the auxiliary strain, electric and magnetic fields through a constant constitutive tensor comprising of elastic, piezoelectric, piezomagnetic, electromagnetic, dielectric permittivities and magnetic permeabilities, evaluated at the crack tip, substituting $\sigma_{i j}^{(2)}=\left(C_{i j k s}^{*}-C_{i j k s}\right) \varepsilon_{k s}^{(2)}-\left(e_{s i j}^{*}-\right.$ $\left.e_{s i j}\right) E_{s}^{(2)}-\left(h_{s i j}^{*}-h_{s i j}\right) H_{s}^{(2)}+C_{i j k s} \varepsilon_{k s}^{(2)}-e_{s i j}$ $E_{s}^{(2)}-h_{s i j} H_{s}^{(2)}, D_{i}^{(2)}=\left(e_{i k s}^{*}-e_{i k s}\right) \varepsilon_{k s}^{(2)}+$ $\left(\kappa_{i s}^{*}-\kappa_{i s}\right) E_{s}^{(2)}+\left(\beta_{i s}^{*}-\beta_{i s}\right) H_{s}^{(2)}+e_{i k s} \varepsilon_{k s}^{(2)}+$ 
$\kappa_{i s} E_{s}^{(2)}+\beta_{i s} H_{s}^{(2)}$, and $B_{i}^{(2)}=\left(h_{i k s}^{*}-h_{i k s}\right) \varepsilon_{k s}^{(2)}+$ $\left(\beta_{i s}^{*}-\beta_{i s}\right) E_{s}^{(2)}+\left(\gamma_{i s}^{*}-\gamma_{i s}\right) H_{s}^{(2)}+h_{i k s} \varepsilon_{k s}^{(2)}+$

$$
\begin{aligned}
& =\lim _{r \rightarrow 0} \int_{\theta} \int_{r} \mathrm{O}\left(r^{-\frac{1}{2}}\right) \mathrm{O}\left(r^{\alpha}\right) \mathrm{O}\left(r^{-\frac{1}{2}}\right) q r d r d \theta \\
& =\lim _{r \rightarrow 0} \mathrm{O}\left(r^{\alpha+1}\right)=0
\end{aligned}
$$

gral of Eq. 56 in the limit $r \rightarrow 0$ becomes,

$$
\begin{aligned}
& \lim _{r \rightarrow 0} \int_{A} \frac{1}{2}\left(\begin{array}{c}
\sigma_{i j}^{(1)} \varepsilon_{i j, 1}^{(2)}-\sigma_{i j, 1}^{(1)} \varepsilon_{i j}^{(2)}+\sigma_{i j}^{(2)} \varepsilon_{i j, 1}^{(1)}-\sigma_{i j, 1}^{(2)} \varepsilon_{i j}^{(1)} \\
-D_{j}^{(1)} E_{j, 1}^{(2)}+D_{j, 1}^{(1)} E_{j}^{(2)}-D_{j}^{(2)} E_{j, 1}^{(1)}+D_{j, 1}^{(2)} E_{j}^{(1)} \\
-B_{j}^{(1)} H_{j, 1}^{(2)}+B_{j, 1}^{(1)} H_{j}^{(2)}-B_{j}^{(2)} H_{j, 1}^{(1)}+B_{j, 1}^{(2)} H_{j}^{(1)}
\end{array}\right) q d A \\
& =\lim _{r \rightarrow 0} \int_{A} \frac{1}{2}\left(\begin{array}{c}
\sigma_{i j}^{(1)} \varepsilon_{i j, 1}^{(2)}-\sigma_{i j, 1}^{(1)} \varepsilon_{i j}^{(2)}+\sigma_{i j}^{(2)} \varepsilon_{i j, 1}^{(1)}-\sigma_{i j, 1}^{(2)} \varepsilon_{i j}^{(1)} \\
-D_{j}^{(1)} E_{j, 1}^{(2)}+D_{j, 1}^{(1)} E_{j}^{(2)}-D_{j}^{(2)} E_{j, 1}^{(1)}+D_{j, 1}^{(2)} E_{j}^{(1)} \\
-B_{j}^{(1)} H_{j, 1}^{(2)}+B_{j, 1}^{(1)} H_{j}^{(2)}-B_{j}^{(2)} H_{j, 1}^{(1)}+B_{j, 1}^{(2)} H_{j}^{(1)}
\end{array}\right) q r d r d \theta \\
& =\lim _{r \rightarrow 0} \int_{\theta} \int_{r}\left(\mathrm{O}(r) \mathrm{O}\left(r^{-\frac{1}{2}}\right) \mathrm{O}\left(r^{-\frac{3}{2}}\right)-\mathrm{O}\left(r^{\alpha}\right) \mathrm{O}\left(r^{-\frac{1}{2}}\right) \mathrm{O}\left(r^{-\frac{1}{2}}\right)\right) q r d r d \theta \\
& =\lim _{r \rightarrow 0} \mathrm{O}(r)=0
\end{aligned}
$$

(ii) Similarly the incompatibility terms in the second integral of Eq. 60 in the limit $r \rightarrow 0$ becomes,

$$
\begin{aligned}
\lim _{r \rightarrow 0} \int_{A}\left(\sigma_{i j}^{(1)}\left(u_{i, j 1}^{(2)}-\varepsilon_{i j, 1}^{(2)}\right)+D_{j}^{(1)}\left(\phi_{, j 1}^{(2)}+E_{j, 1}^{(2)}\right)\right. \\
\left.\quad+B_{j}^{(1)}\left(\varphi_{, j 1}^{(2)}+H_{j, 1}^{(2)}\right)\right) q d A \\
=\lim _{r \rightarrow 0} \int_{A}\left(\sigma_{i j}^{(1)}\left(u_{i, j 1}^{(2)}-\varepsilon_{i j, 1}^{(2)}\right)+D_{j}^{(1)}\left(\phi_{, j 1}^{(2)}+E_{j, 1}^{(2)}\right)\right. \\
\left.\quad+B_{j}^{(1)}\left(\varphi_{, j 1}^{(2)}+H_{j, 1}^{(2)}\right)\right) q r d r d \theta \\
=\lim _{r \rightarrow 0} \int_{\theta} \int \mathrm{O}(r) \mathrm{O}\left(r^{-\frac{1}{2}}\right) \mathrm{O}\left(r^{-\frac{3}{2}}\right) q r d r d \theta \\
=\lim _{r \rightarrow 0} \mathrm{O}(r)=0
\end{aligned}
$$

(iii) Rest of the terms in the second integral of Eq. 60 in the limit $r \rightarrow 0$ becomes, (iv) Following similar procedure as given Paulino and Kim 2003, in the limit $r \rightarrow 0$ the nonequilibrium terms in the integrand of the second integral in Eq. 64 becomes,

$$
\begin{aligned}
\lim _{r \rightarrow 0} \int_{A}\left(\sigma_{i j, j}^{(2)} u_{i, 1}^{(1)}+D_{j, j}^{(2)} \phi_{, 1}^{(1)}+B_{j, j}^{(2)} \varphi_{, 1}^{(1)}\right) q d A \\
=\lim _{r \rightarrow 0} \int_{A}\left(\sigma_{i j, j}^{(2)} u_{i, 1}^{(1)}+D_{j, j}^{(2)} \phi_{, 1}^{(1)}+B_{j, j}^{(2)} \varphi_{, 1}^{(1)}\right) \\
\quad \times q \operatorname{rdrd} \theta \\
=\lim _{r \rightarrow 0} \int_{\theta} \int_{r} \mathrm{O}(r) \mathrm{O}\left(r^{-\frac{3}{2}}\right) \mathrm{O}\left(r^{-\frac{1}{2}}\right) q r d r d \theta \\
=\lim _{r \rightarrow 0} \mathrm{O}(r)=0
\end{aligned}
$$

In the limit $r \rightarrow 0$, rest of the terms in the second integral of Eq. 64 becomes as given in (iii). Thus

$$
\begin{gathered}
\lim _{r \rightarrow 0} \int_{A}\left(\begin{array}{c}
-\varepsilon_{i j}^{(1)} C_{i j k l, 1} \varepsilon_{k l}^{(2)}+E_{n}^{(1)} \kappa_{n m, 1} E_{m}^{(2)}+H_{n}^{(1)} \gamma_{n m, 1} H_{m}^{(2)} \\
+\left(\varepsilon_{k l}^{(2)} e_{n k l, 1} E_{n}^{(1)}+\varepsilon_{k l}^{(1)} e_{n k l, 1} E_{n}^{(2)}\right) \\
+\left(\varepsilon_{k l}^{(2)} h_{n k l, 1} H_{n}^{(1)}+\varepsilon_{k l}^{(1)} h_{n k l, 1} H_{n}^{(2)}\right) \\
+\left(E_{n}^{(2)} \beta_{n m, 1} H_{m}^{(1)}+E_{n}^{(1)} \beta_{n m, 1} H_{m}^{(2)}\right)
\end{array}\right) q d A \\
=\lim _{r \rightarrow 0} \int_{A}^{(1)}\left(\begin{array}{c}
-\varepsilon_{i j}^{(1)} C_{i j k l, 1} \varepsilon_{k l}^{(2)}+E_{n}^{(1)} \kappa_{n m, 1} E_{m}^{(2)}+H_{n}^{(1)} \gamma_{n m, 1} H_{m}^{(2)} \\
+\left(\varepsilon_{k l}^{(2)} e_{n k l, 1} E_{n}^{(1)}+\varepsilon_{k l}^{(1)} e_{n k l, 1} E_{n}^{(2)}\right) \\
+\left(\varepsilon_{k l}^{(2)} h_{n k l, 1} H_{n}^{(1)}+\varepsilon_{k l}^{(1)} h_{n k l, 1} H_{n}^{(2)}\right) \\
+\left(E_{n}^{(2)} \beta_{n m, 1} H_{m}^{(1)}+E_{n}^{(1)} \beta_{n m, 1} H_{m}^{(2)}\right)
\end{array}\right) q r d r d \theta \\
\end{gathered}
$$


all the proposed interaction integrals for non-homogeneous magnetoelectroelastic materials in Eqs. 56, 60 and 64 are well posed as the limit $r \rightarrow 0$ exists.

It should be pointed out that the above changes in appendix do not practically affect the results presented in the paper, because the numerical results are based on Eqs. 56, 60 and 64 and not on their proof of existence. 